\title{
Vibrotactile Information for Intuitive Speed Regulation
}

\author{
Jani Lylykangas ${ }^{1}$, Veikko Surakka ${ }^{1}$, Jussi Rantala ${ }^{1}$, Jukka Raisamo ${ }^{1}$, \\ Roope Raisamo ${ }^{1}$, and Esa Tuulari ${ }^{2}$ \\ ${ }^{1}$ Tampere Unit for Computer-Human Interaction \\ (TAUCHI) \\ Department of Computer Sciences \\ University of Tampere \\ Kanslerinrinne 1, FIN-33014 Tampere, Finland \\ Tel.: +358 335518565 \\ Emails: \{firstname.lastname\}@cs.uta.fi \\ ${ }^{2}$ Polar Electro Ltd. \\ Professorintie 5 \\ FIN-90440 Kempele, Finland \\ Tel.: +358 85202100 \\ Email: esa.tuulari@polar.fi
}

\begin{abstract}
The present aim was to investigate if controlled vibrotactile stimulation can be used to inform users on how to regulate their behavior. 36 stimuli were varied by frequency modulation (i.e., ascending, constant, and descending), duration (i.e., 500, 1750, and $3000 \mathrm{~ms}$ ), waveform (i.e., sine and sawtooth), and body location (i.e., wrist and chest), and presented to 12 participants. The participants were to evaluate without any training the meaning of each presented stimuli using three response options: 'accelerate your speed', 'keep your speed constant', and 'decelerate your speed'. Participants rated also how emotionally pleasant and arousing the different stimulations were. The results showed that the stimuli were predominantly perceived analogously with the vibration frequency modulation. The best stimuli represented 'accelerate your speed', 'keep your speed constant', and 'decelerate your speed' information in accuracies of 88,100 , and $79 \%$, respectively. Stimulations were experienced as more pleasant in the wrist compared to the chest location. Both ascending and descending stimulations were rated as more arousing than stimuli with constant frequency. Our results suggest that tactile stimulation could be used in real life mobile applications, for example, in sports to inform the users on how they should regulate their performance.
\end{abstract}

\section{Categories and Subject Descriptors}

H.5.2 [Information Interfaces and Presentation]: User Interfaces - Evaluation/methodology, Haptic I/O.

\section{General Terms}

Measurement, Performance, Design, Experimentation, Human Factors.

\section{Keywords}

Tactile feedback, tactile icons, speed regulation, non-visual interaction.

\section{INTRODUCTION}

There are a growing number of human-technology interaction (HTI) applications that utilize measurement and interpretation of

(C) The Author 2009.

Published by the British Computer Society bodily functions for improving human physical performance. For instance, the effects of using heart rate biofeedback in guiding physical training have been proved beneficial [7]. The interpreted measurement data can currently be mediated continuously and in real time to the users in order to help them to regulate their behavior and performance throughout the physical performance like running.

Mobile sports computers have nowadays many guiding features such as functionalities for heart rate monitoring, timekeeping and navigation. Traditionally these devices provide the needed information for the user during a physical exercise (e.g., when the heart rate exceeds the set target range) using auditory alerts and/or visual feedback for adjusting the level of the performance (e.g., to slow down the speed). The full exploitation of visual and auditory feedback modalities can, however, be restricted if the user has low vision or low hearing ability.

Sometimes the access to the needed information can be prevented by situational or context related reasons as well. Taking a running exercise in urban surroundings is a rather extreme example of a context where situational constrains are faced, but at a same time, it is a common situation for the growing number of sports computers users. The noise of the traffic and the need for focusing one's attention to the environment can prevent the efficient use of both auditory and visual outputs. On the other hand, in more peaceful situations auditory feedback can be considered as disturbing either by the user or by other people around. Listening to information via earphones might be effective but not always recommendable because it could prevent the user from processing important environmental information such as hearing sounds of approaching vehicles. Due to potential safety risks, in many U.S. marathon events, for example, the entrants are prohibited from using earphones for music listening [1].

It is clear that alternative ways to mediate information on the status of human bodily functioning would be welcome. One promising solution is to extend the bandwidth by adding other modalities to mediate information to the users of mobile technology. Haptics is a modality that is gaining increasing interest supported by the latest scientific results and improved technology. The research in the field of haptics focuses on the use of the sense of touch, active touching, and haptic feedback in HTI. Tactile expression refers to technologies that are able to give tactile stimulation by using certain predefined parameters. By their sense of touch humans can interpret the tactile expressions provided by, for example, a mobile device.

Intuitively, it is apparent that presenting information via the sense of touch (i.e., haptic feedback) would have several advantages compared to the use of visual and auditory modalities in the mobile contexts of use. It would free the user from sharing the visual and auditory attention between the environment and the messaging device. In this sense the potential of having haptic 
feedback seems a promising way to proceed in developing safer and user-friendlier devices for mobile users.

In fact, there is evidence that the tactile sense can be used successfully in proving guidance during a physical exercise [9]. In another study, van Erp et al. [6] provided motion co-ordination information on the angles of the knees and the back during a set of multiple rowing exercises. Feedback was given either by visual information on the computer screen or by analogous vibrotactile information with actuators located on the back and on the knee. Co-ordination accuracies with vibrotactile and visual feedback groups were equally good, but interestingly, the vibrotactile group showed decrease on the heart rate along the experiment while in the visual group the heart rates were increasing. The authors interpreted the results so that more effort was required to maintain the optimal performance by following visual than vibrotactile information during the exercise.

Recent studies have found that tactile expression can also be used for producing iconic information by using predefined parameters. Brewster and Brown [3] introduced a concept of tactile icons (i.e., Tactons) as a communication method to complement graphical and auditory feedback at the user interface. Tactile icons are structured, abstract messages that can be used for non-visual information presentation. The authors suggest that distinguishable and identifiable information can be encoded by manipulating, for example, frequency, amplitude, duration, rhythm, and spatial location of the tactile icons. Brown et al. [4] created 9 twodimensional tactile icons informing about the type of a phone alert (i.e., three different rhythms) and the priority of the alert (i.e., three different amplitude modulations within each rhythm) using advanced actuator technology. The results showed that the icons were recognized correctly with the accuracy of $71 \%$. Later on, Brown and Kaaresoja [5] were able to reach equal recognition rates using a standard vibration actuator of a mobile phone in creating similar iconic information.

We note, however, that in these studies it has been typical that the recognition rates for iconic information were achieved by teaching the meaning of the icons for the participants in a separate learning session before the actual experiment. Of course, it would be ideal if the tactile expression could be designed so that they can be interpreted effortlessly, or even intuitively. For this reason also studies that do not coach experimental participants are needed.

Our aim was to first investigate the possibilities of providing intuitive tactile information in carefully controlled laboratory settings with immobile participants. Only after the case that we would find that easily interpretable or intuitive feedback seems possible, we should proceed to more realistic settings and mobile users. Earlier research has shown that physical movement can attenuate the recognition accuracy of low-amplitude vibrotactile stimuli [8], and therefore it was reasonable to use relatively high stimulus amplitude for ensuring that the same stimuli could be usable also in the future studies, where physical exercise was planned to be present.

It was hypothesized that different vibration frequency profiles (i.e., dynamic and constant) could have the potential to intuitively provide the users with analogous information about the regulation of the speed. We were encouraged by the results of our pilot studies which indicated that ascending, constant, and descending frequencies could be associated with speed acceleration, constant speed, and speed deceleration, respectively. Wrist and chest were chosen for the reason that they are the locations of the main components of commonly used sports monitors (i.e., wrist unit and heart rate monitoring chest belt) and thus natural platforms for embedding haptic technology. The other stimulus parameters were selected for investigating the effects on different waveforms and stimulus durations.

Tactile expression has also been found to be associated to human emotions [10]. As the stimuli should be noticeable but preferably not too much irritating, we also wanted to measure the emotion related subjective experiences evoked by the stimuli. Earlier research has shown that the use of dimensional affective space can be effective in tracking down the emotion related responses of the participants. We decided to use subjective rating scales for arousal and pleasantness, which have been previously used, for example, by Bradley and Lang [2] and Salminen et al. [10].

\section{METHODS}

\subsection{Participants}

Twelve naïve and voluntary male participants took part in the experiment (mean age 22.8 years, range 19-31 years). By their self reports eleven were right-handed and one was left-handed. They all reported that they had normal sense of touch.

\subsection{Apparatus}

Two Engineering Acoustic Inc. (http://www.eaiinfo.com/) C-2 vibrotactile voice coil actuators were used to present Windows Waveform (WAV) audio files through a sound card of a PC computer. The diameter of the C-2 actuator was $3.05 \mathrm{~cm}(1.2$ "), and the diameter of the vibrating skin contactor area was $0.76 \mathrm{~cm}$ (0.3"). Stimuli were amplified with a Stage Line ${ }^{\circledR}$ STA 1508 eight-channel amplifier. The actuators were attached to the wrist with elastic wristband and to the chest with elastic and adjustable Polar $^{\mathbb{B}}$ WearLink ${ }^{\mathrm{TM}}$ heart rate monitoring chest belt (Figure 1).

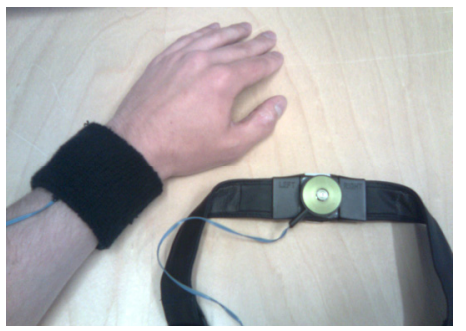

Figure 1. Wrist actuator attached under a wristband and backside view of chest actuator attachment to a chest belt.

E-Prime $^{(} 2.0$ Professional experiment generator software [11] was used to control the stimulus presentation and to collect the data. Responses were given with a modified number key section of a standard computer keyboard. Three response buttons were set in a vertical layout. The uppermost button was labeled with an arrow pointing upward $(\pi)$, the middle button was labeled with an arrow pointing sideward $(\rightarrow)$, and the undermost button was labeled with an arrow pointing downward $(\searrow)$. Green experiment initiation button was located on the left side of the middle button. Unnecessary buttons were removed.

\subsection{Stimuli}

18 different stimuli were used to present vibrotactile information on two body sites (i.e., wrist and chest). The stimuli were varied by frequency modulation, duration, and waveform of the signal. The stimuli were built upon two different waveforms (i.e., sine and sawtooth wave). These waveforms were selected based on both $\mathrm{C}-2$ actuator recommendations on using sine wave stimuli (see http://www.eaiinfo.com/) and pilot tests. The pilot tests showed that sawtooth stimuli performed better in differentiating the ascending, constant, and descending frequency modulations as 
compared to the use of square wave stimuli. Three different durations of the stimuli were used (i.e., 500, 1750, and $3000 \mathrm{~ms}$ ) providing clearly distinctive temporal time frame for the stimulation. The frequency profiles of the stimuli were manipulated using three different types of frequency modulations (i.e., ascending, constant, and descending frequency). For ascending stimuli the frequency was modulated linearly from 50 to $300 \mathrm{~Hz}$ (Figure 2a). The frequency for the constant stimuli was kept at $175 \mathrm{~Hz}$ (Figure 2b). For descending stimuli the frequency was modulated linearly from 300 to $50 \mathrm{~Hz}$ (Figure 2c). The amplitude and phase of the signal were not altered.

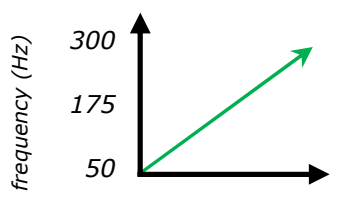

a)

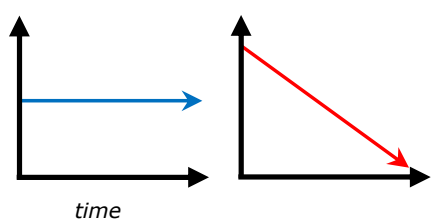

b) c)
Figure 2. Frequency modulations used for ascending (a), constant (b), and descending (c) stimuli.

Audacity $^{\circledR}$ audio editor and recorder software version 1.2.6 (http://audacity.sourceforge.net/) and Pure Data real-time audio synthesizer software version 0.40 .3 (http://puredata.info/) were used in creating the stimuli. All the stimuli were 16-bit mono WAV files with $44.1 \mathrm{kHz}$ sampling frequency. Stimulus amplitude was adjusted to $1.49 \mathrm{~V}(\mathrm{AC})$ for both channels (i.e., for wrist and chest) by measuring the voltage from the amplifier outputs with a Fluke ${ }^{\circledR} 87 \mathrm{~V}$ True RMS multimeter while the actuators were playing a constant $250 \mathrm{~Hz}$ sine wave calibration stimulus.

\subsection{Procedure}

First, the laboratory and the equipment were introduced to the participant. Then he was seated and the actuators were attached on top of the wrist of the non-dominant hand and to the chest. The actuator in the chest was located in the sagittal plane of the thorax. In order to avoid unwanted vibration resonance with the skeleton, the actuator was attached below the breastbone. To ensure this the participant was instructed to put his thumb under his breastbone and then to adjust the height of the chest belt so that the actuator was right under the thumb. The participant was informed that the heart rate data was not measured during the experiment, although the chest belt used was originally from a heart rate monitor. The non-dominant hand was laid on a table on top of a foam plastic armrest and the other hand was put on top of the response buttons. The participant was instructed to hold the non-dominant hand still and to keep the gaze on the display during the experiment. In order to block the mechanical sound of the actuators, the participant listened pink noise (i.e., $1 / f$ noise) via a hearing protector headset at a comfortable sound level.

There were three sessions: stimulus familiarization session, cognitive evaluation session, and emotional rating session. The order of the sessions was fixed. The emotional rating session consisted of two sub-sessions (i.e., A for pleasantness ratings and $\mathrm{B}$ for arousal ratings). The order of the sub-sessions was counterbalanced between the participants so that half of them started with sub-session A and then continued with sub-session B. The other half performed the sub-sessions in reverse order. Each of the sessions and sub-sessions consisted of two separate blocks. In block A, the stimuli were presented to the wrist and in block B, the stimuli were presented to the chest. The performance order of the blocks within each session and sub-session was counterbalanced between the participants so that half of them started with block A and then continued with block B. The other half performed the blocks $\mathrm{A}$ and $\mathrm{B}$ in reverse order. The participant was allowed to take a short break between the sessions, sub-sessions and blocks. Finally, there was a structured but informal post-experimental interview, where the participant was asked questions to get additional information, for example, on the response strategy and about opinions regarding the difficultness of the experimental tasks. It took an average of one hour for conducting the whole experiment.

\subsubsection{Stimulus Familiarization Session}

The purpose of the first session was to allow the participant to familiarize himself in feeling the vibrotactile stimulations in the two body sites and to initiate him into the stimulus space used in the experiment. The task was simply to sit still and to feel the stimuli. After giving the instructions, the participant was allowed to start the first session by pressing the experiment initiation button. Then all the 18 stimuli were presented sequentially in a randomized order for the participant with an interstimulus interval of $3500 \mathrm{~ms}$. In block A, the stimuli were presented to the wrist, and in block B, the stimuli were presented to the chest. Thus, there were a total of 36 trials. The response buttons were covered for preventing of giving any hints of the experimental task in the upcoming cognitive evaluation session.

\subsubsection{Cognitive Evaluation Session}

In the beginning of the second session the experimenter uncovered the response buttons and explained the purpose of the experiment. The participant was instructed to imagine that the computer was giving him instructions to either accelerate his speed, to keep his speed constant, or to decelerate his speed on the basis of the stimuli presented in the first session.

First, the participant performed three practice trials in order to train the response technique. In the practice session, three 1000 ms long practice stimuli (constant 50, 175, and $300 \mathrm{~Hz}$ square wave vibration) were presented one at a time in a randomized order simultaneously to the wrist and to the chest. The practice stimuli were not presented in the actual experiment.

After the practice trials, the experimenter left the room and the participant was allowed to initiate the first trial by pushing the experiment initiation button. In a trial, a text "Next stimulus" appeared on the display for $1500 \mathrm{~ms}$. Then the text disappeared and a fixation point $(+)$ appeared on the center of the display for $1500 \mathrm{~ms}$. The display was blank for $500 \mathrm{~ms}$ before the stimulus onset and during the stimulus presentation. Immediately after the stimulus offset, the response options appeared on the display, and the participant was able to answer. The task was to evaluate the meaning of the stimulus between three response options. The uppermost button was to be pushed for 'Accelerate your speed' responses, the middle button was to be pushed for 'Keep your speed constant' responses, and the undermost button was to be pushed for 'Decelerate your speed' responses. The participant was instructed to give the response as quickly as possible and to rely on the first impression. After the press of a response button there was a $500 \mathrm{~ms}$ interval and then a new trial was initiated automatically.

Each of the 18 stimuli was presented twice to the wrist (block A) and twice to the chest (block B) in a randomized order. Thus, there were a total of 72 trials.

\subsubsection{Emotional Rating Session}

In the third session the task was to rate the subjective experiences evoked by the stimuli using two emotion-related nine-point bipolar scales varying from -4 to +4 . In sub-session A, ratings 
were asked for the scale of pleasantness of the stimulus varying from unpleasant to pleasant (i.e., how unpleasant or pleasant the stimulus felt). In sub-session B, ratings were asked for the scale of arousal of the stimulus varying from relaxed to aroused (i.e., how relaxed or aroused the participant felt during the stimulus). On both of the scales, 0 represented a neutral experience (i.e., neither unpleasant nor pleasant and neither relaxed nor aroused). Ratings were given using a computer keyboard with nine keys labeled from -4 to +4 .

In the beginning of both sub-sessions the participant performed three practice trials in order to practice giving the ratings. In the practice trials, three practice stimuli were rated one at a time. The practice stimuli were presented simultaneously to the wrist and to the chest. They were not presented in the actual experiment.

After the practice trials, the experimenter left the room and the participant was allowed to start rating the experimental stimuli. The participant was able to decide when to initiate a trial by pushing a space bar on the keyboard. Then a fixation point appeared on the center of the display for $1500 \mathrm{~ms}$. The display was blank for $1500 \mathrm{~ms}$ before the stimulus onset and during the stimulus presentation. Immediately after the stimulus offset, the rating scale appeared on the display, and the participant was able to respond. After giving the response there was a $500 \mathrm{~ms}$ interval and then the participant could initiate a new trial.

In both sub-sessions each of the 18 stimuli were presented once to the wrist (block A) and once to the chest (block B) in a randomized order. Thus, there were a total of 72 trials.

\subsection{Data analysis}

The experiment was a within-subject $3 \times 3 \times 2 \times 2$ (frequency modulation $\times$ duration $\times$ waveform $\times$ location) design. Repeated measures analysis of variance (ANOVA) was used for statistical analysis. If the sphericity assumption of the data was violated, Greenhouse-Geisser corrected degrees of freedom were used to validate the $F$ statistic. Bonferroni corrected pairwise $t$-tests were used for post hoc tests.

\section{RESULTS}

Figures 3-8 show the mean responses and standard error of the means (S.E.M.s) in the cognitive evaluation session. Figures 9-12 present the mean responses and S.E.M.s in the emotional rating session.

\subsection{Cognitive Evaluations}

\subsection{1 'Accelerate your speed' Responses}

For the 'Accelerate your speed' responses (see Figures 3 and 4), a four-way $3 \times 3 \times 2 \times 2$ (frequency modulation $\times$ duration $\times$ waveform $\times$ location) ANOVA showed a statistically significant main effect for the frequency modulation $F(2,22)=26.0$, p < 0.001 and for the waveform $F(1,11)=29.1, \mathrm{p}<0.001$. The main effects for the duration and the location were not statistically significant.

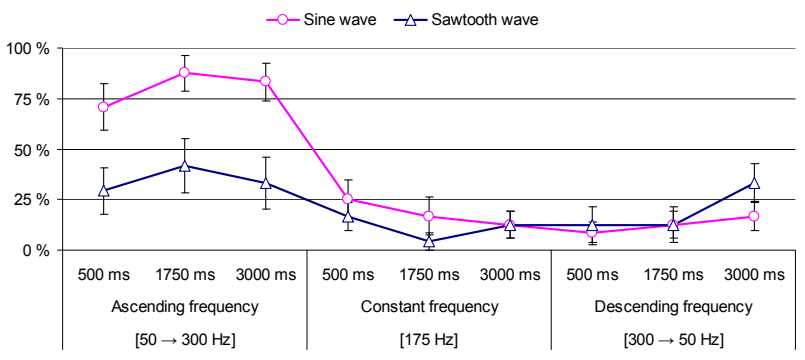

Figure 3. Mean percentages and S.E.M.s for

'Accelerate your speed' responses in wrist by frequency modulation, duration, and waveform.

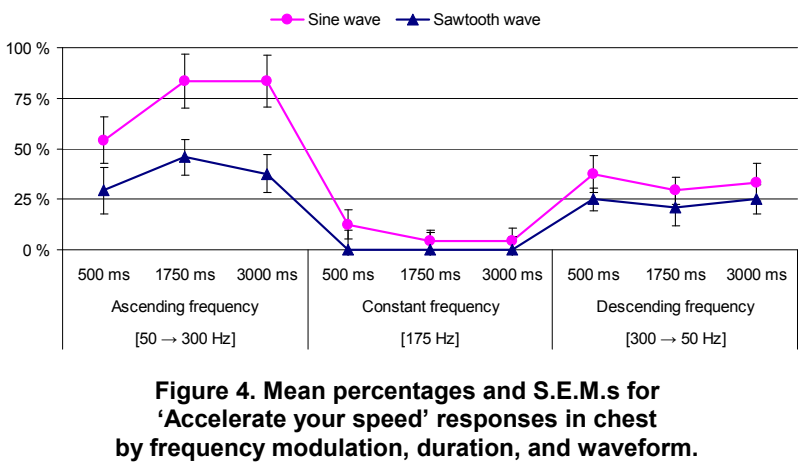

There was a statistically significant interaction effect between the frequency modulation and the waveform $F(2,22)=15.1, \mathrm{p}<$ 0.001 , between the frequency modulation and the location $F(2$, $22)=9.3, p \leq 0.001$, and between the frequency modulation and the duration $F(4,44)=3.6, p<0.05$. There were no other statistically significant interactions of the main effects.

To analyze the interaction effects in more detail, four separate one-way ANOVAs were performed. One-way ANOVAs revealed a significant effect of the frequency modulation $F(2,22)=26.0, p$ $<0.001$ and the waveform $F(1,11)=29.1, p<0.001$, but the effects of the duration and the location were not statistically significant.

Post hoc pairwise comparisons showed that the participants evaluated the meaning of the stimuli with ascending frequency more frequently as 'Accelerate your speed' than the stimuli with constant frequency $M D=0.47, p<0.001$ and descending frequency $M D=0.34, p<0.01$. In addition, the meaning of the stimuli with descending frequency was evaluated more frequently as 'Accelerate your speed' than the stimuli with constant frequency $M D=0.13, p<0.01$. The sine wave stimuli were evaluated more frequently as 'Accelerate your speed' than the sawtooth wave stimuli $M D=0.16, p<0.001$. The other pairwise comparisons were not statistically significant.

\subsection{2 'Keep your speed constant' Responses}

For the 'Keep your speed constant' responses (see Figures 5 and 6 ), a four-way $3 \times 3 \times 2 \times 2$ (frequency modulation $\times$ duration $\times$ waveform $\times$ location) ANOVA showed a statistically significant main effect for the frequency modulation $F(2,22)=88.4, p<$ 0.001 , for the duration $F(2,22)=6.6, p<0.05$, and for the waveform $F(1,11)=44.1, p<0.001$. The main effect for the location was not statistically significant. 


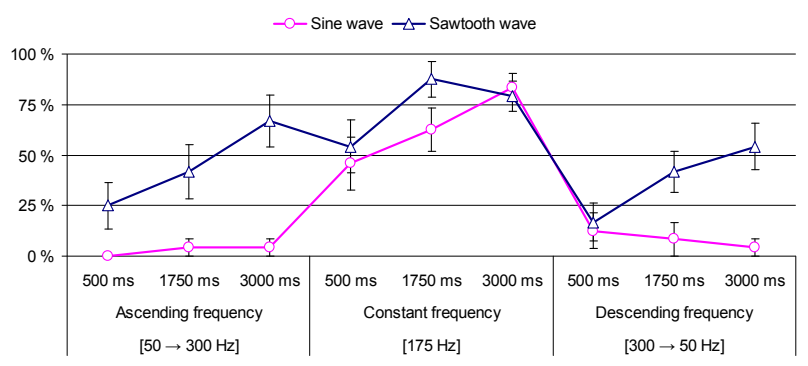

Figure 5. Mean percentages and S.E.M.s for 'Keep your speed constant' responses in wrist by frequency modulation, duration, and waveform.

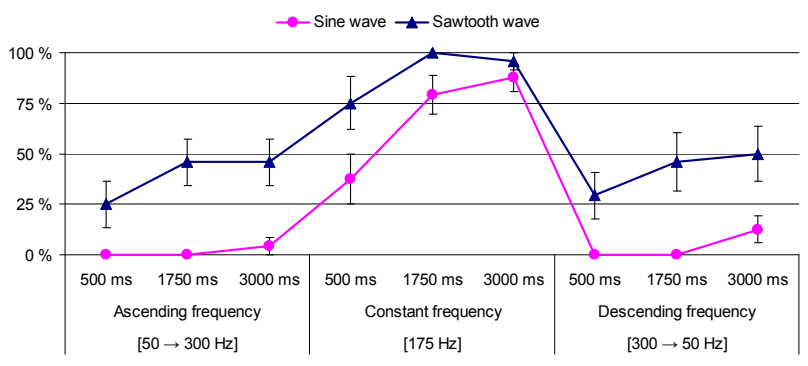

Figure 6. Mean percentages and S.E.M.s for

'Keep your speed constant' responses in chest by frequency modulation, duration, and waveform.

There was a statistically significant interaction effect between the frequency modulation and the waveform $F(2,22)=7.5, p<0.01$. There were no other statistically significant interactions of the main effects.

To analyze the interaction effect in more detail, two separate oneway ANOVAs were performed. One-way ANOVAs revealed a significant effect of the frequency modulation $F(2,22)=88.4, p<$ 0.001 , and the waveform $F(1,11)=44.1, p<0.001$.

Post hoc pairwise comparisons showed that the participants evaluated the stimuli with constant frequency more often as 'Keep your speed constant' than the stimuli with ascending frequency $M D=0.52, p<0.001$ and descending frequency $M D=0.51, p<$ 0.001 . The sawtooth wave stimuli were evaluated more often as 'Keep your speed constant' than the sine wave stimuli $M D=0.30$, $p<0.001$. The other pairwise comparisons were not statistically significant.

\subsection{3 'Decelerate your speed' Responses}

For the 'Decelerate your speed' responses (see Figures 7 and 8), a four-way $3 \times 3 \times 2 \times 2$ (frequency modulation $\times$ duration $\times$ waveform $\times$ location) ANOVA showed a statistically significant main effect for the frequency modulation $F(2,22)=26.7, p<$ 0.001 , for the waveform $F(1,11)=11.4, p<0.01$, and for the duration $F(2,22)=4.9, p<0.05$. The main effect for the location was not statistically significant.

There was a statistically significant interaction effect between the frequency modulation, the duration, and the waveform $F(4,44)=$ $4.1, p<0.01$. In addition, there was a statistically significant interaction effect between the frequency modulation and the waveform $F(2,22)=10.1, p<0.01$, between the frequency modulation and the location $F(2,22)=4.2, p<0.05$, and between the duration and the waveform $F(2,22)=4.1, p<0.05$. There were no other statistically significant interactions of the main effects.

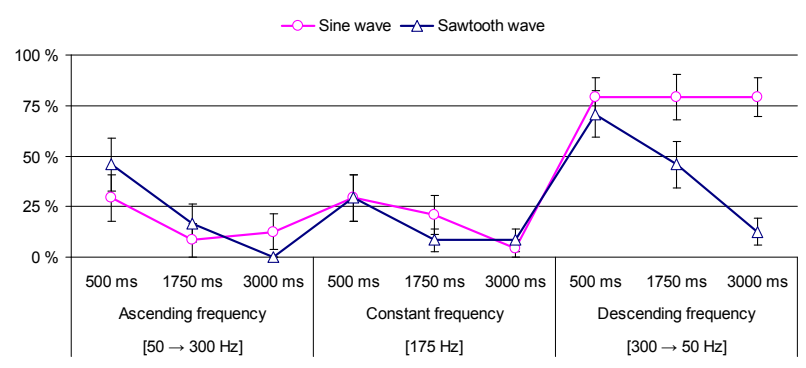

Figure 7. Mean percentages and S.E.M.s for 'Decelerate your speed' responses in wrist by frequency modulation, duration, and waveform.

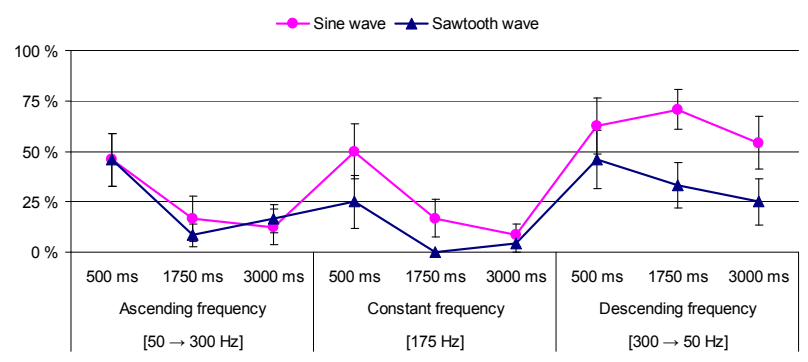

Figure 8. Mean percentages and S.E.M.s for 'Decelerate your speed' responses in chest by frequency modulation, duration, and waveform.

To analyze the interaction effects in more detail, three separate two-way ANOVAs and three separate one-way ANOVAs were performed. Two-way ANOVAs revealed the significant interactions between the frequency modulation and the waveform $=F(2,22)=10.1, p<0.01$, and between the duration and the waveform $=F(2,22)=4.1, p<0.05$, but not between the frequency modulation and the duration. One-way ANOVAs revealed a significant effect of the frequency modulation $F(2,22)$ $=26.7, p<0.001$ and the waveform $F(1,11)=11.4, p<0.01$, but the effect of the location was not statistically significant.

Post hoc pairwise comparisons showed that the participants evaluated the stimuli with descending frequency more frequently as 'Decelerate your speed' than the stimuli with ascending frequency $M D=0.33, p<0.01$ and constant frequency $M D=$ $0.38, p<0.001$. The sine wave stimuli were evaluated more frequently as 'Decelerate your speed' than the sawtooth wave stimuli $M D=0.13, p<0.01$. The other pairwise comparisons were not statistically significant.

\subsection{Emotional Ratings}

\subsubsection{Pleasantness}

For the ratings of pleasantness (see Figures 9 and 10) a four-way $3 \times 3 \times 2 \times 2$ (frequency modulation $\times$ duration $\times$ waveform $\times$ location) ANOVA showed a statistically significant main effect for the duration $F(2,22)=5.8, p<0.05$ and for the location $F(1$, $11)=7.4, p<0.05$. The main effects for the frequency modulation and the waveform were not statistically significant, and there were no statistically significant interactions of the main effects. 


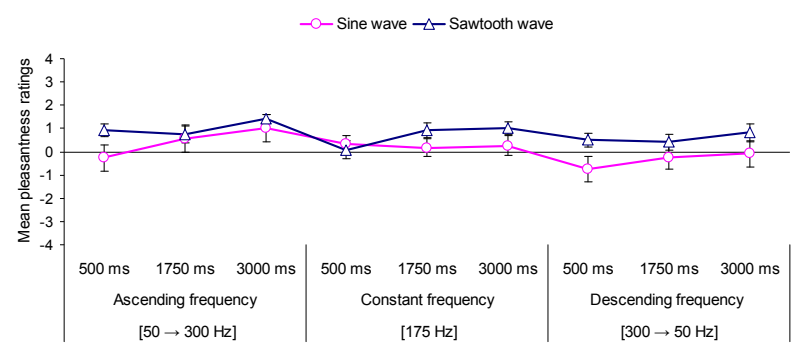

Figure 9. Mean ratings and S.E.M.s for pleasantness of the stimuli in wrist by frequency modulation, duration, and waveform.

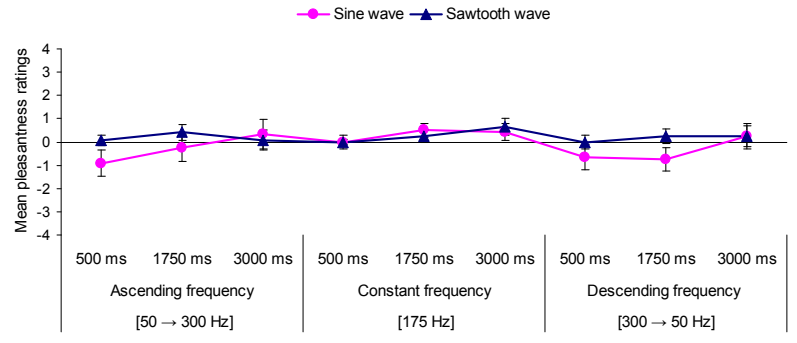

Figure 10. Mean ratings and S.E.M.s for pleasantness of the stimuli in chest by frequency modulation, duration, and waveform.

Post hoc pairwise comparisons showed that the participants rated the $3000 \mathrm{~ms}$ long stimuli as significantly more pleasant than the $1750 \mathrm{~ms}$ long stimuli $M D=0.29, p<0.05$. In addition, the stimuli were rated as significantly more pleasant in the wrist than in the chest $M D=0.38, p<0.05$. The other pairwise comparisons were not statistically significant.

\subsubsection{Arousal}

For the ratings of arousal (see Figures 11 and 12) a four-way $3 \times 3$ $\times 2 \times 2$ (frequency modulation $\times$ duration $\times$ waveform $\times$ location) ANOVA showed a statistically significant main effect for the frequency modulation $F(2,22)=59.6, p<0.001$, for the duration $F(2,22)=5.8, p<0.05$, and for the waveform $F(1,11)=44.2, p$ $<0.001$. The main effect of the location was not statistically significant.

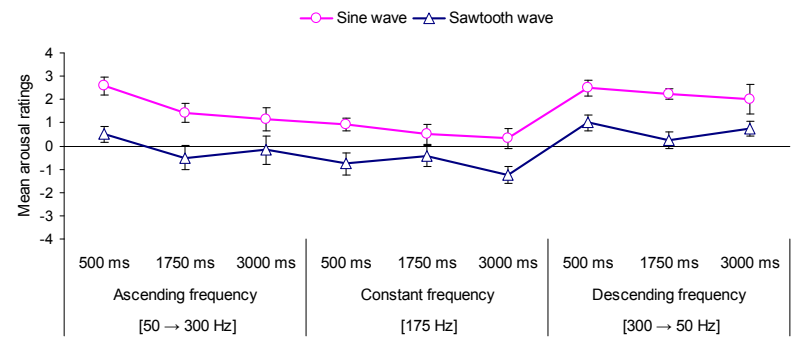

Figure 11. Mean ratings and S.E.M.s for arousal of the stimuli in wrist by frequency modulation, duration, and waveform.

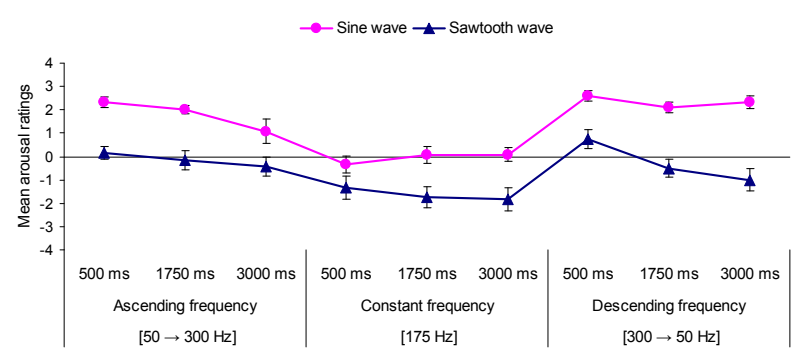

Figure 12. Mean ratings and S.E.M.s for arousal of the stimuli in chest by frequency modulation, duration, and waveform.
There was a statistically significant interaction effect between frequency modulation and the location $F(2,22)=4.0, p<0.05$. There were no other statistically significant interactions of the main effects.

To analyze the interaction effect in more detail, two separate oneway ANOVAs were performed. One-way ANOVAs revealed a significant effect of the frequency modulation $F(2,22)=59.6, p<$ 0.001 , but the effect of the location was not statistically significant.

Post hoc pairwise comparisons showed that the participants felt less aroused during the stimuli with constant frequency than during the stimuli with ascending frequency $M D=-1.31, p<$ 0.001 and descending frequency $M D=-1.73, p<0.001$. In addition, the participants felt less aroused during the sawtooth wave stimuli than during the sine wave stimuli $M D=-1.81, p<$ 0.001 . The other pairwise comparisons were not statistically significant.

\subsection{Post-experimental Interviews}

The most central findings in the interviews were the following. Five of the total of twelve participants thought that the task in evaluating the stimuli between the response options was easy rather than difficult, and one participant was caught between the two options. The rest thought that the task was difficult rather than easy, but two of them said that the task became easier during the experiment.

When asked about the used response strategies, none of the participants reported giving answers purely on random basis. The most common response strategy was based on the analogy with the perceived frequency modulation. Ten participants reported that they were able to recognize three types of frequency dynamics, and that they based their answers mainly on that. In addition, two of them mentioned that they also used stimulus duration in associating the shortest stimuli with 'Decelerate your speed' responses. Two participants based their responses mainly on the stimulus duration.

Whereas eight participants considered the wrist as a more sensitive location for feeling the stimuli, the chest was regarded as more sensitive by the rest. When asked about their preference between the two locations, nine reported rather feeling the stimuli in the wrist than in the chest, and three voted for the chest. None of the participants reported hearing the mechanical sound of the stimuli.

\section{DISCUSSION AND SUMMARY}

Our results showed that our hypothesis was well supported, as the meanings of the stimuli were predominantly perceived and evaluated analogously with the vibration frequency modulation without teaching. The best performing stimuli could represent information for accelerating the speed, for keeping the speed constant, and for decelerating the speed in accuracies of 88,100 , and $79 \%$, respectively. These accuracies were clearly above the $33.3 \%$ chance level. In general, sine wave stimuli outperformed sawtooth wave stimuli in representing 'Accelerate your speed' and 'Decelerate your speed' information, but at the same time sawtooth wave stimuli functioned better in representing 'Keep your speed constant' information compared to the sine wave stimuli. Differences between different stimulus durations were not statistically significant, although there was a tendency for associating the shortest (i.e., $500 \mathrm{~ms}$ ) stimuli with 'Decelerate your speed' responses and longer (i.e., 1750 and $3000 \mathrm{~ms}$ ) stimuli with 'Keep your speed constant' responses. There were no 
statistically significant differences in the stimulus evaluations between the wrist and chest locations. The empirical results were supported by post-experimental interviews, where the majority of the participants reported that they had created a response strategy which was in analogy with the perceived frequency modulation of the stimuli. Most of the participants thought that the task felt rather difficult, especially in the very beginning, but that at the same time, as the experiment proceeded, it became easier.

To summarize the results from the statistical analysis of the cognitive evaluation data (see Figures 3-8), we would recommend using an ascending frequency sine wave stimulus with long duration for presenting information on speed acceleration. On the contrary, a constant frequency sawtooth wave stimulus with medium duration should be used for presenting information on keeping the speed constant. Descending frequency sine wave stimuli with short duration would perform well in presenting information on speed deceleration.

Emotional ratings (see Figures 9-12) for arousal and pleasantness showed that stimuli with constant frequency were evaluated as less arousing than stimuli with ascending and descending frequency. Although, the sine wave stimuli were evaluated as more arousing than the sawtooth wave stimuli, there were no differences in the pleasantness ratings between the two waveforms. This is interesting because in the earlier study by Salminen et al. [10], tactile stimuli rated as arousing, were also rated as unpleasant, and respectively, relaxing stimuli were rated as pleasant. In stimulus discrimination tasks of the same study, more arousing stimuli were also reacted faster and they appeared to be more distinguishable than less arousing stimuli.

In the current experiment, the participants rated the longest stimuli (i.e., $3000 \mathrm{~ms}$ ) as more pleasant than the stimuli with shorter durations (i.e., 500 and $1750 \mathrm{~ms}$ ). In general, all stimuli were also evaluated as more pleasant in the wrist than in the chest. This result was supported by the post-experimental interviews where the majority of the participants preferred wrist more than chest. Further, most participants reported that their wrist was more sensitive for feeling the stimuli than their chest. Even so, there seemed to be individual differences in the touch sensitivity between the two locations as one of the participants verbalized that some stimuli felt 'too thrilling' in the chest. Generally speaking, our stimuli were, however, rated as fairly neutral in respect of their pleasantness, even though relatively high stimulus amplitudes were used in order to be able to provide detectable stimuli for the real life contexts and physically active users.

The results were promising because they indicated that it is possible to provide interpretable information to guide the user's performance without loading the user's visual and auditory channels. We argue that we were able to convey information intuitively in the sense that in the particular context we did set for our participants, the stimuli could be interpreted naturally and coherently. This happened despite the fact that the meaning of the stimuli was not taught for the participants at any point of the experiment. An interesting detail was that one of the stimuli (i.e., constant frequency / $1750 \mathrm{~ms} /$ sawtooth wave) was evaluated in the chest as 'Keep your speed constant' in the rate of one hundred percent.

Based on the current results it seems that in respect to the idea of being effortlessly interpretable or intuitive, our stimulations functioned in quite promising way. Given that the factual intuitiveness of the stimuli requires further research, we are executing a follow-up study using two groups with both female and male participants. In one group the participants are first trained the meaning of the different stimuli in the beginning of the experiment. Similarly to the present study, the other group is not taught or coached in any way. By comparing the response rates between these groups, we will be able to get a better understanding of the intuitiveness of the stimuli. We can also analyze if there are any differences between the responses of women and men. Finally, we are planning to move on outside the laboratory and test how the system would work in a realistic sports computer use. This way we will be able to evaluate if tactile expression works better as a support to visual and auditory feedback or would it be the case that tactile expression is fully sufficient on its own.

\section{ACKNOWLEDGMENTS}

This research was a part of the Mobile Haptics project funded by the Finnish Funding Agency for Technology and Innovation (Tekes), decision 40120/08.

\section{REFERENCES}

[1] Blackburn, J. C., Hersh, B., Kleeman, G., and Podkaminer, B. (Eds.) 2009. USA Track \& Field 2009 Competition Rules. Washington St., Indianapolis.

[2] Bradley, M. and Lang, P. J. 1994. Measuring emotion: The self-assessment manikin and the semantic differential. Journal of Behavioral Therapy and \& Experimental Psychiatry, 25, 1, 49-59.

[3] Brewster, S. and Brown, L. M. 2004. Tactons: structured tactile messages for non-visual information display. In Proceedings of the Fifth Conference on Australasian User interface - Volume 28 (Dunedin, New Zealand). A. Cockburn, Ed. ACM International Conference Proceeding Series, vol. 53. Australian Computer Society, Darlinghurst, Australia, 15-23.

[4] Brown, L. M., Brewster, S. A., and Purchase, H.C. 2005. A first investigation into the effectiveness of Tactons. In Proceedings of the First Joint Eurohaptics Conference and Symposium on Haptic Interfaces for Virtual Environment and Teleoperator Systems (Pisa, Italy, March 18 - 20, 2005). WorldHaptics 2005. IEEE, 167-176. DOI= http://dx.doi.org/10.1109/WHC.2005.6

[5] Brown, L. M. and Kaaresoja, T. 2006. Feel who's talking: using tactons for mobile phone alerts. In CHI '06 Extended Abstracts on Human Factors in Computing Systems (Montréal, Québec, Canada, April 22 - 27, 2006). CHI '06. ACM, New York, NY, 604-609. DOI= http://doi.acm.org/10.1145/1125451.1125577

[6] van Erp, J.B.F., Saturday, I., and Jansen, C. 2006. Application of tactile displays in sports: Where to, how and when to move. In Proceedings of the Eurohaptics International conference (Paris, France, July 3-6, 2006). EuroHaptics 2006. IEEE, 90-95.

[7] Hautala, A., Mäkikallio, T., Kiviniemi, A., Laukkanen, R., Nissilä, S., Huikuri, H., and Tulppo, M. 2004. Heart rate dynamics after controlled training followed by a home-based exercise program. European Journal of Applied Physiology, 92, 3, July, 289-297.

[8] Pakkanen, T., Lylykangas, J., Raisamo, J., Raisamo, R., Salminen, K., Rantala, J., and Surakka, V. 2008. Perception of low-amplitude haptic stimuli when biking. In Proceedings of the 10th international Conference on Multimodal interfaces (Chania, Crete, Greece, October 20 - 22, 2008). IMCI '08. ACM, New York, NY, 281-284. DOI= http://doi.acm.org/10.1145/1452392.1452449 
[9] Pertovaara, A., Kemppainen, P., and Leppänen, H. 1992 Lowered cutaneous sensitivity to nonpainful electrical stimulation during isometric exercise in humans. Experimental Brain Research, 89, 2, 447-452.

[10] Salminen, K., Surakka, V., Lylykangas, J., Raisamo, J., Saarinen, R., Raisamo, R., Rantala, J., and Evreinov, G. 2008. Emotional and behavioral responses to haptic stimulation. In Proceeding of the Twenty-Sixth Annual SIGCHI Conference on Human Factors in Computing
Systems (Florence, Italy, April 05 - 10, 2008). CHI '08.

ACM, New York, NY, 1555-1562. DOI=

http://doi.acm.org/10.1145/1357054.1357298

[11] Schneider, W., Eschman, A., and Zuccolotto, A. 2002. EPrime User's Guide. Psychology Software Tools, Inc, Pittsburgh. 